\title{
Editorial
}

\section{Applications of Spectroscopy in Environmental Monitoring of Gases and Aerosols}

\author{
Arnaud Cuisset, ${ }^{1}$ Dean S. Venables, ${ }^{2}$ Xiaoming Gao, ${ }^{3}$ and Hassen Aroui ${ }^{4}$ \\ ${ }^{1}$ Laboratoire de Physico-Chimie de l'Atmosphère, Université du Littoral Côte d'Opale, 59140 Dunkerque, France \\ ${ }^{2}$ Department of Chemistry, National University of Ireland, University College of Cork, Cork, Ireland \\ ${ }^{3}$ Anhui Institute of Optics and Fine Mechanics, Chinese Academy of Sciences, Hefei 230031, China \\ ${ }^{4}$ Laboratoire de Dynamique Moléculaire et Matériaux Photoniques, Université de Tunis, \\ Ecole Nationale Supérieure d'Ingénieurs de Tunis, 5 Avenue Taha Hussein, 1008 Tunis, Tunisia
}

Correspondence should be addressed to Arnaud Cuisset; arnaud.cuisset@univ-littoral.fr

Received 7 May 2016; Accepted 8 May 2016

Copyright (C) 2016 Arnaud Cuisset et al. This is an open access article distributed under the Creative Commons Attribution License, which permits unrestricted use, distribution, and reproduction in any medium, provided the original work is properly cited.

Spectroscopy has long been the most important tool for investigating the earth's atmosphere. Indeed, it is worth noting that spectroscopy is the only method that can be used for remote observations of the atmosphere. Remote measurements are indispensable given both the spatial heterogeneity of the atmosphere's chemical composition and physical properties, as well as the financial and technical difficulties (or outright inability) to probe the atmosphere directly with in situ sensors of any type. In situ spectroscopic methods are also widely used in atmospheric studies, including measuring short-lived reactive species that drive the chemistry of the atmosphere, isotopologue concentrations, and the optical properties of particles, which strongly alter radiative forcing and influence climate. The information provided by such spectroscopic methods is critical to determining air quality, identifying emissions of air pollutants, quantifying the magnitude and fluxes of climate forcing agents, ensuring compliance with regulations, and informing environmental policies at national and international levels.

Despite its long history, new developments in atmospheric spectroscopy continue to be made both on the instrumental side and on the analysis of spectra. On one hand, on-going instrumental advances continue to be driven by new technologies in light sources, sample environments, and detectors; on the other hand, the ability and need to collect increasingly detailed spatial and temporal information continue to promote the importance of novel spectral analysis strategies. So, along with the emergence of new techniques, even apparently mature spectroscopic techniques continue to evolve in both scope and performance.
This special issue focuses on several examples of developments in applying spectroscopy to atmospheric and air quality measurements. The articles feature a range of techniques covering wavelengths from the ultraviolet through to the mid-infrared spectral regions using both active and passive light sources. This broad spectral range is suited to various measurement targets, ranging from water vapour and the major anthropogenic greenhouse gases, carbon dioxide and methane, to the classical air pollutants, ozone and nitrogen dioxide. Although most applications focus on direct atmospheric observations, the applications of spectroscopy to air quality in mines and industrial plume monitoring are also considered. Technological solutions such as acoustic microresonators and dual field-of-view lidar allow us to improve sensitivity detection limits for gas detection and improved coverage of atmospheric aerosols at near and far ranges. It is also notable how strongly spectral analysis features in the papers. This is to be expected given the increasing spatial information from various approaches and the importance of remote observations. The articles underline these considerations with new inversion algorithms and calibration methods for trace gas retrievals and hyperspectral imaging in the long-wave infrared. Taken together, these studies indicate the breadth of methods, the range of applications, and the ongoing development in spectroscopy as an essential tool for understanding the atmosphere.

Arnaud Cuisset Dean S. Venables Xiaoming Gao Hassen Aroui 

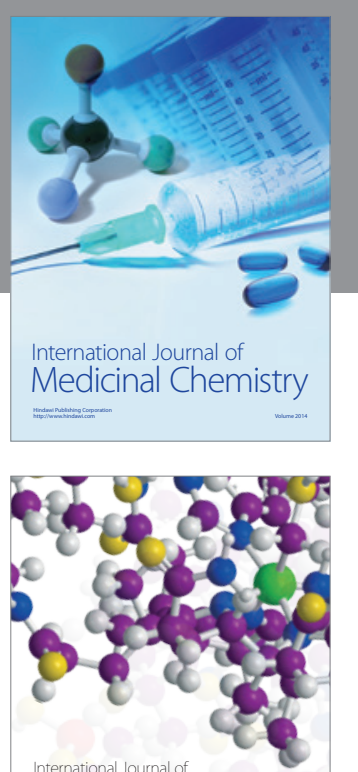

Carbohydrate Chemistry

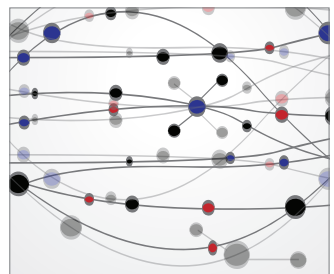

The Scientific World Journal
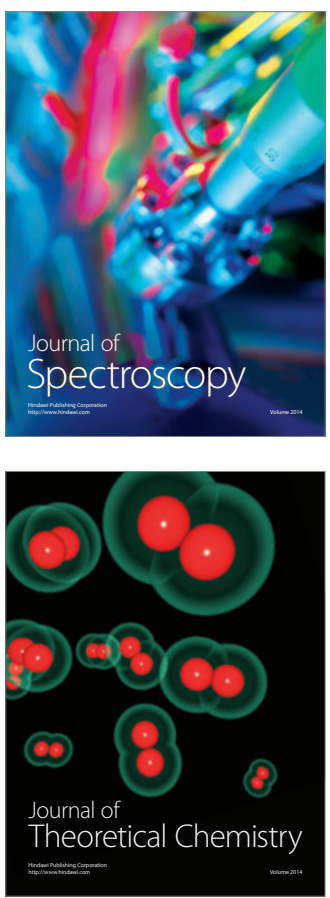
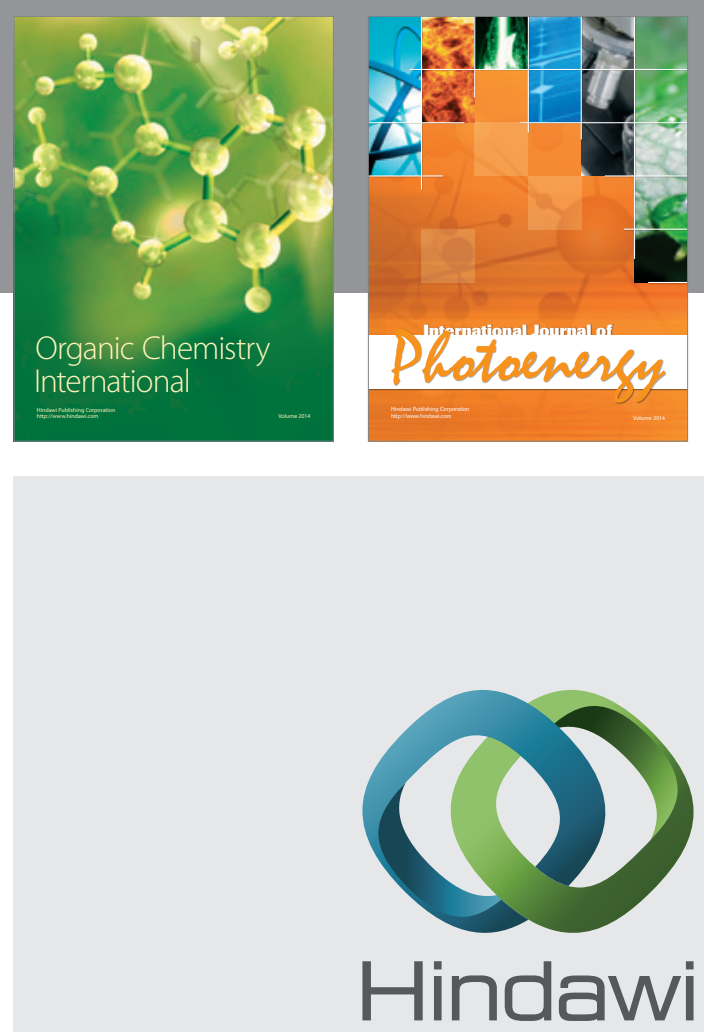

Submit your manuscripts at

http://www.hindawi.com

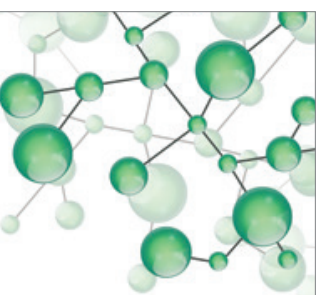

International Journal of

Inorganic Chemistry

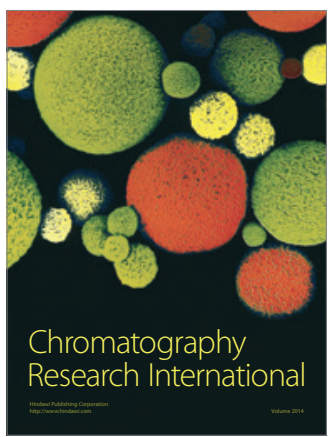

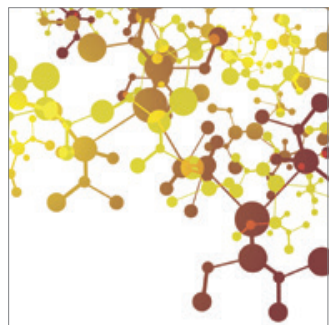

Applied Chemistry
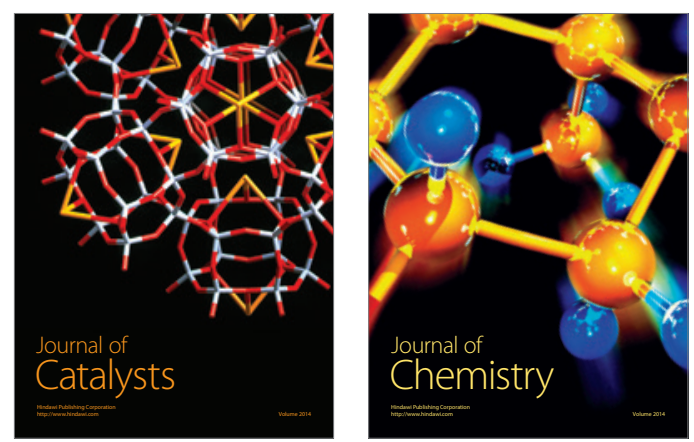
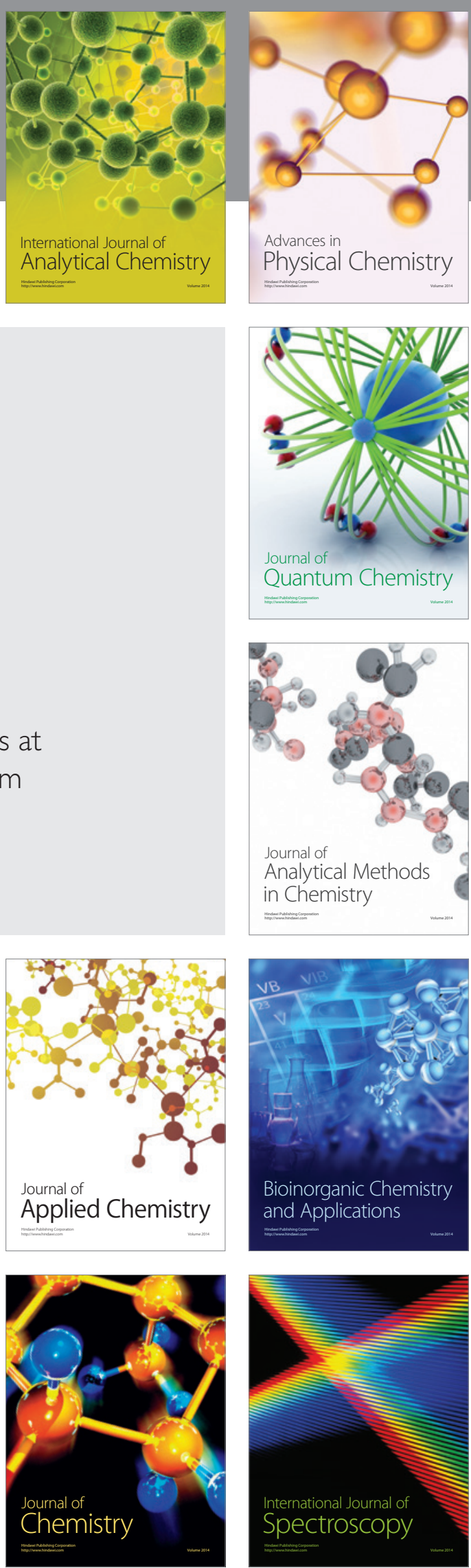